\title{
Éléments finis étendus pour la modélisation des structures soudées par points
}

\section{Eléments enrichis pour points de soudure}

\author{
Pierre Burry* — Habibou Maitournam** — G. Billotey*** \\ *Direction de la Recherche et de l'Innovation Automobile, Peugeot Citroën \\ Automobiles, Route de Gisy F-78943 Vélizy Villacoublay cedex \\ ** Laboratoire de Mécanique des Solides, Ecole Polytechnique, F-91128 Palaiseau \\ cedex \\ *** Ecole Polytechnique
}

\begin{abstract}
RÉSUMÉ. Cet article présente un élément de coque bilinéaire enrichi, adapté à la modélisation des tôles soudées par points de soudure électrique, et implémenté sous Abaqus. L'élément de base est un élément de Simo-Rifaï en petits déplacements et en élasticité linéaire. La modélisation proposée des points de soudure s'inspire des méthodes de modélisation d'inclusion dans un milieu infini par éléments finis étendus. Le point de soudure est considéré comme quasi ponctuel dans le milieu continu $2 D$ de la tôle; les fonctions d'enrichissement de l'espace d'approximation des déplacements représentent les modes de déformation locaux des tôles autour des points de soudure, et permettent de représenter correctement les déformées de tôles assemblées sans avoir à mailler les points de soudure. Des exemples démontrent la possibilité de calculer des assemblages de tôles maillées indépendamment.
\end{abstract}

ABSTRACT. This paper presents an enriched bilinear quadrilateral thin shell element for spot welded structure modelling implemented in an Abaqus user element. The base element is a Simo-Rifaï quadrilateral. The present treatment of spot welds is inspired from the inclusion problem treatment in the extended finite element framework. Each spot weld is considered as a quasi punctual detail in the steel sheet $2 \mathrm{D}$ continuous medium; the enrichment functions to the displacement approximation space are chosen to represent accurately the local deformation modes near the spot weld, and give an acceptable representation of the deformed assembled steel sheets without requiring an explicit meshing of spot welds. Numerical examples demonstrate the method capability to model spot welded structures from independently obtained steel sheets meshes.

MOTS-CLÉS : Soudage par point, XFEM, éléments coques, assemblages, Abaqus.

KEYWORDS: Spot welding, XFEM, shell element, assembly modelling, Abaqus.

\section{Introduction : limites de la modélisation actuelle des points de soudure}

Le point de soudure électrique (PSE) est un assemblage qui se réalise sans apport de matière, par fusion des tôles mises en contact. C'est aujourd'hui la 
technique d'assemblage par excellence des structures automobiles. Les méthodes de modélisation existantes des tôles soudées par points, par exemple chez Peugeot Citroën Automobiles, reposent sur un critère formulé sur le torseur d'effort transitant par le PSE. Le calcul de ce torseur nécessite un maillage explicite des PSE par l'utilisateur. La méthode impose de mailler simultanément les deux tôles assemblées et de créer des maillages compatibles de la position des centres des PSE.

Pour automatiser le maillage des structures soudées, on peut créer des liaisons cinématiques ponctuelles entre les tôles. Ce choix conduit à une surévaluation de la raideur de la structure : les tôles de plusieurs mètres sont généralement maillées avec une taille de maille de 15 à $20 \mathrm{~mm}$, et un tel maillage est incapable de représenter les modes de déformation ponctuels autour des PSE. Pour obtenir une raideur de structure acceptable, on peut remplacer les liaisons cinématiques rigides par des ressorts (Beniguel, 2002) de raideur variable. Cela n'élimine pas complètement les manipulations de maillages et ne permet pas de faire un calcul de fatigue prédictif.

Cet article présente une alternative pour la modélisation des PSE : un élément fini étendu de coque. A l'échelle d'une structure automobile, quelques mètres, un point de soudure de quelques millimètres peut être considéré comme ponctuel. L'idée directrice est de créer des liaisons cinématiques entre les tôles assemblées et d'enrichir les fonctions de formes des éléments autour des points de soudure pour représenter correctement les modes de déformation locaux.

\section{Elément de base}

L'élément de base choisi est un élément de coque bilinéaire à quatre nœuds similaire au S4 d'Abaqus (Abaqus Inc, 2003). La modélisation du comportement plan de cet élément est basée sur la formulation mixte proposée par Simo et Rifaï (Simo et al, 1990) dans le cadre de l'élasticité plane, pour éviter le verrouillage en cisaillement pour les problèmes dominés par la flexion dans le plan des coques. Le cisaillement transverse est traité par discrete colocation constraint (Zienkiewicz et $a l, 2000)$ pour éviter le verrouillage en cisaillement pour les problèmes dominés par la flexion. La raideur en rotation perpendiculaire au plan de la coque, le degré de liberté de drill, est traité par un terme de pénalisation non physique [1] dans lequel $\omega_{z}$ est la rotation d'ensemble et $\theta_{z}$ les fonctions de forme de drill aux nœuds. Ce choix rend impossible le calcul d'une raideur physique de torsion des PSE sur un maillage coque.

$$
\int_{\Omega} \alpha E t^{3}\left(\theta_{z}-\omega_{z}\right) d \Omega
$$




\section{Elément de coque enrichi}

\subsection{Idée générale}

Dans le cadre des éléments finis étendus, et autres méthodes de partition de l'unité (I. Babuska et al, 1996), on suppose préexistante une modélisation par éléments finis posée sur un domaine global $\Omega g$. Cette modélisation est supposée satisfaisante en dehors d'une zone $\Omega l$. L'idée générale est d'ajouter aux fonctions de formes $N_{I}$ de chaque nœud $I$ du domaine $\Omega_{l}$ les fonctions de formes produit $N_{I} X$ des fonctions de formes du nœud $I$ avec une fonction représentant une solution particulière du problème. Le choix des fonctions $X$ dépend de la physique du problème : pour la modélisation d'une pointe de fissure, il est pertinent de choisir les solutions de la mécanique linéaire de la rupture en milieu infini (N. Moës et al, 1999). Suivant (N. Sukumar et al, 2002), pour intégrer ces éléments ainsi créés à un code de calcul existant, on crée des éléments utilisateur avec des nœuds supplémentaires dont les degrés de liberté correspondent aux degrés de liberté nodaux enrichis. Un maillage fin est créé pour visualiser les déplacements obtenus avec les éléments enrichis.

\subsection{Choix des enrichissement : flexion locale}

Dans le cas des structures assemblées par PSE, le problème est de représenter correctement les déformations localisées des tôles autour des points de soudure. Dans un premier temps, on ne considérera que les flexions des tôles autour d'un PSE chargé en moment, $M_{x}$ ou $M_{y}$, dans le plan de la tôle. Les fonctions d'enrichissement choisies sont les solutions du problème de la plaque carrée percée encastrée au centre et en appui simple sur la périphérie. Ces fonctions ont été obtenues numériquement sur un maillage de référence (voir figure 1), puis tabulées.

Le mode de déformation principal de la tôle autour du PSE est un mode de flexion, et donc on a choisi de n'enrichir que les champs de rotation. Pour chaque direction de moment, $M_{x}$ ou $M_{y}$, on obtient deux fonctions d'enrichissement qu'on utilise indépendamment : le champ de rotation autour de $e_{x}$ et celui autour de $e_{y}$.
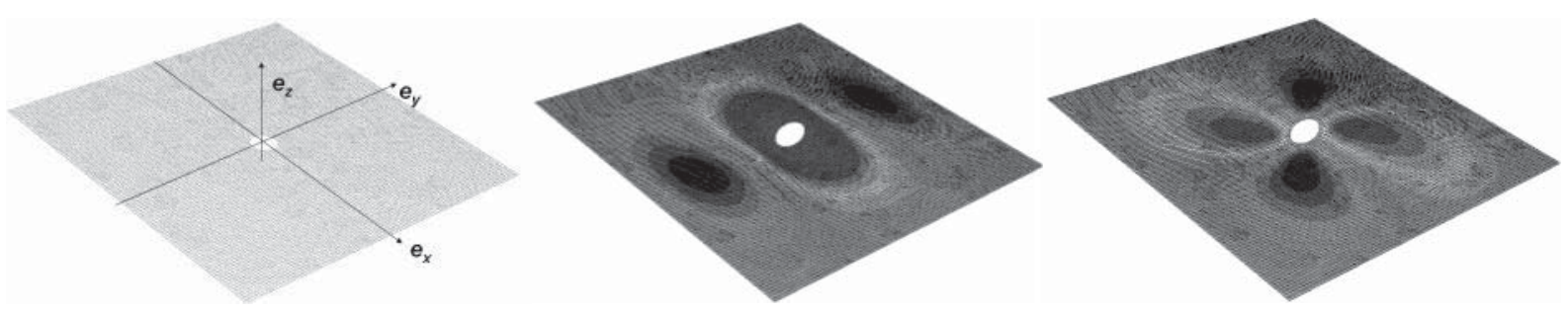

Figure 1. Maillage de référence, fonctions d'enrichissement des champs de rotation d'axe $e_{x}$ et $e_{y}$. Le repère local du point de soudure coüncide avec le repère global. Les champs de rotations autour de $e_{x}$ et $e_{y}$ sont utilisés comme des enrichissements indépendants. 
Les fonctions d'enrichissement $X(x, y)$ sont tabulées dans le repère local du PSE, voir figure 1. Pour «plaquer» cette solution particulière sur la surface de coque, on crée un difféomorphisme $\Phi$ du plan vers la surface de la coque. Lors de la phase de pré traitement, on crée un champ nodal qui associe à chaque nœud enrichi $I$ la valeur de $\Phi^{-1}\left(x_{I}\right)$. Au cours du calcul élémentaire, $\Phi$ est interpolé à partir de ses valeurs nodales. La fonction d'enrichissement en coordonnées paramétriques est définie par: $X\left(\sum_{I} \Phi^{-1}\left(x_{I}\right) N_{I}\right)$. Seuls les nœuds à une distance inférieure à 3 fois le diamètre du point de soudure sont enrichis.

\subsection{Liaison cinématique entre deux tôles}

Pour relier deux tôles, on a besoin de relier la cinématique des points coïncidents en tôles supérieure et inférieure. Il faut créer une relation linéaire entre les degrés de liberté $(d d l)$ classiques et enrichis de l'élément de la tôle supérieure et ceux de l'éléments de la tôle inférieure. Comme on ne connaît pas les valeurs des fonctions de formes enrichies au centre du PSE avant le calcul de la matrice de rigidité, on a choisi de traiter directement ce problème au cours du calcul par une approche par multiplicateurs de Lagrange. Cela revient à ajouter trois termes à la fonctionnelle [2] du problème variationnel :

$$
\Pi^{*}\left(u, \lambda_{1}, \lambda_{2}, \mu\right)=\Pi+\lambda_{1} L_{1}(u)+\lambda_{2} L_{2}(u)+\mu\left(\lambda_{1}+\lambda_{2}\right)
$$

$L_{1}$ et $L_{2}$ sont les deux formes linéaires des déplacements des points 1 de l'élément de la tôle supérieure et 2 de l'élément de la tôle inférieure que l'on veut égaler. $\lambda_{1}$ et $\lambda_{2}$ sont les efforts de réaction aux points 1 et $2 ; \mu$ est le déplacement relatif des points 1 et 2 .

Concrètement cette stratégie revient à modifier la matrice $K$ des éléments concernés en rajoutant un (ou plusieurs selon le nombre de relations linéaires à imposer) $d d l$ supplémentaire. La nouvelle matrice élémentaire [3] s'écrit alors:

$$
K^{*}=\left(\begin{array}{cc}
K & L^{\prime} \\
L & 0
\end{array}\right)
$$

On relie enfin les degrés de liberté supplémentaires $\lambda_{1}$ et $\lambda_{2}$ par une relation linéaire $\lambda_{1}+\lambda_{2}=0$ qui sera également gérée par multiplicateur de Lagrange par Abaqus.

\subsection{Intégration numérique}

Pour les termes de comportement dans le plan, la méthode d'intégration retenue est une simple quadrature de gauss à $5 \times 5$ points. En effet, les fonctions d'enrichissement retenues sont assez régulières, et une rapide étude de convergence a montré que 5 points suffisaient. Les termes de cisaillement transverse de l'élément classique sont traités par discrete colocation constraint avec 2 fois 2 points d'intégration. L'enrichissement choisi ne porte que sur les degrés de liberté de 
rotation. Les conditions nécessaires de stabilité sont toujours vérifiées. Dans le cas d'un enrichissement avec une fonction de déplacement hors plan (par exemple si on utilise la solution de la plaque en appui sous chargement vertical), il faut rajouter un point d'intégration pour les termes de cisaillement hors plan pour s'assurer que le nombre de points de collocation est égal au nombre d'équations.

\subsection{Une seule tôle enrichie : patch test}

Cette partie ne prétend pas présenter les résultats permettant de valider l'élément enrichi ainsi formulé, mais simplement des patchs tests élémentaires et les premiers résultats de calculs effectués. Le PSE est au centre de la plaque, on impose un déplacement ${ }^{1}$ unitaire en rotation autour de $e_{x}$. On remarque en particulier (voir les champs de rotation en figures 3,4) que les flexions sont un peu mieux représentées sur le maillage enrichi. On ne retrouve pas la solution de référence sur le calcul XFEM car la zone d'enrichissement est limitée autour du PSE.

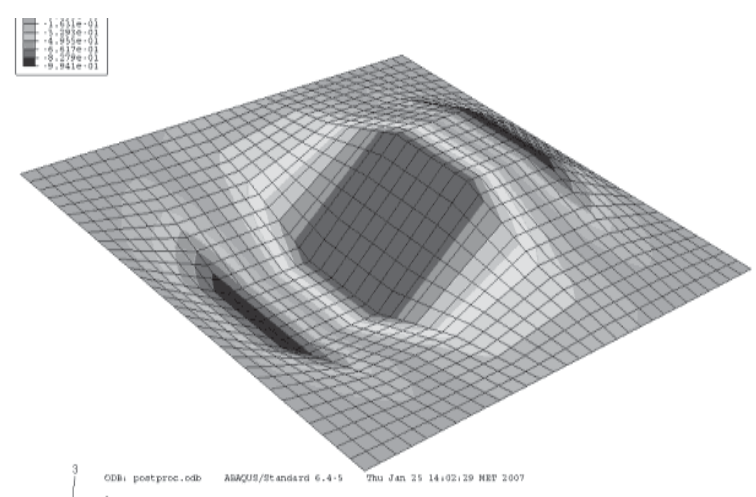

Figure 3. Résultats Abaqus $S 4$

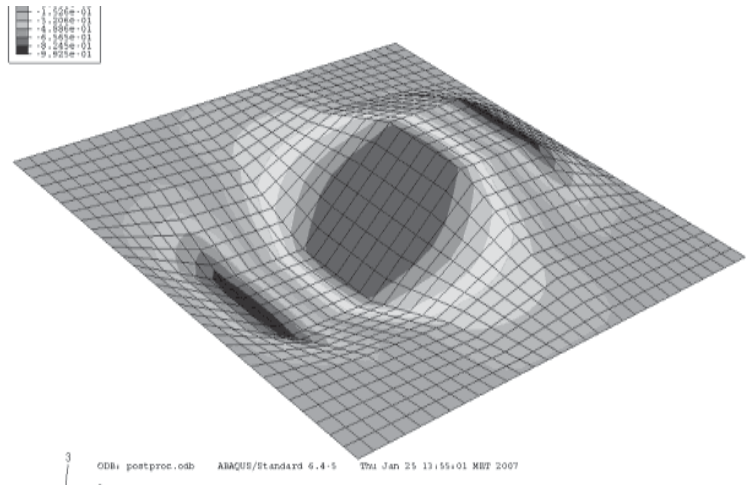

Figure 4. Résultats XFEM

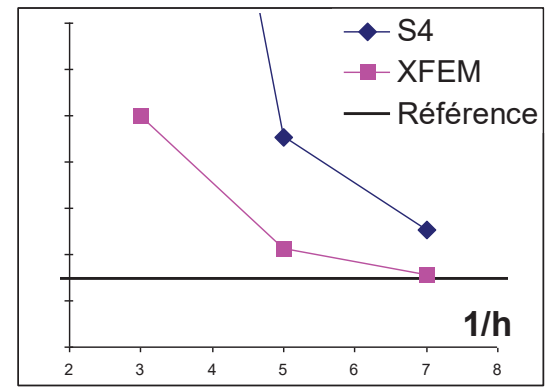

Figure 5. convergence en raideur
La réponse en raideur est représentée figure 5. On remarque que lorsqu'il n'y a plus que 3 éléments pour mailler la plaque $(16 \mathrm{~mm}$ de paramètre de maille, ce qui est déjà relativement fin pour une voiture complète) les éléments finis classiques verrouillent en cisaillement : c'était attendu car les conditions aux limites interdisent tout autre mode de déformation à certains éléments. Les éléments finis enrichis fournissent une meilleure évaluation de la raideur sur cet exemple simple.

\footnotetext{
${ }^{1}$ Le déplacement est imposé sur les degrés de libertés lagrangiens en question au paragraphe 3.3. Techniquement, on utilise une carte *cload (chargement ponctuel !) pour imposer un déplacement, et on lit les efforts de réactions dans les sorties de déplacements.
} 


\section{Conclusion et perspectives}

Nous avons présenté un élément fini de coque à 4 nœuds enrichi adapté à la modélisation des structures soudées par points de soudure électrique. L'élément permet de calculer une raideur de structure plus réaliste même sur un maillage grossier, en incorporant des déformations locales de la tôle autour des points de soudure, voir exemple figure 3. Cet élément permet de représenter un point de soudure entre deux maillages indépendants et incompatibles.

Le principal intérêt de cette modélisation est la possibilité de représenter un PSE sur un maillage prédéfini. La suite de notre travail va maintenant porter sur la représentation de la raideur en torsion des points de soudure.

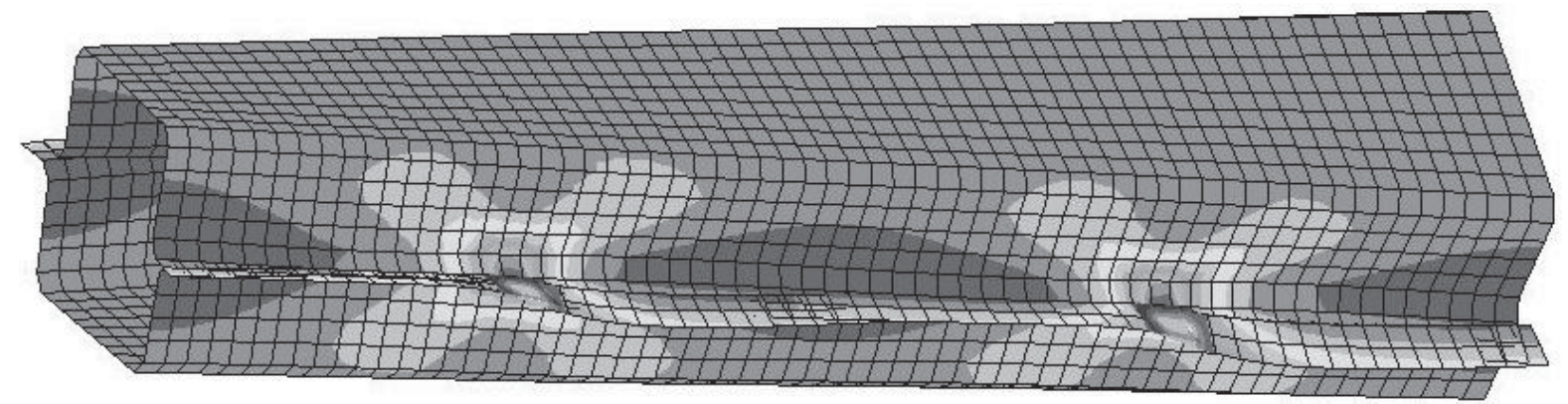

Figure 6. Cisaillement sur un «double oméga » soudé par 4 PSE, sollicité en torsion par la modélisation présentée.

\section{Bibliographie}

Abaqus theory manual, Abaqus Inc., Abaqus 6.4, 2003.

Beniguel J.-F., Modélisation du comportement mécanique des assemblages de structures soudées, Thèse de doctorat, Ecole Normale Supérieure de Cachan, 2002.

Melenk J. M., Babuška I., « The partition of unity finite element method: Basic theory and applications ", Computer methods in applied mechanics and engineering, vol. 139, 1996, p. 289-314.

Moës N., Dolbow J., Belytschko T, «A Finite Element method for crack growth without remeshing », Internationnal Journal for numerical methods in engineering, vol. 46, 1999, p. 131-150.

Simo J. C., Rifaï M. S, «A Class of mixed assumed strain methods and the method of incompatible modes ", Internationnal Journal for numerical methods in engineering, vol. 29, 1990, p. 1595-1638.

Sukumar N., Prévost J.-H., "Modeling quasistatic crack growth with the extended finite element method Part I: Computer implementation », Internationnal Journal of solids and structures, vol. 40, 2003, p. 7513-7537.

Zienkiewicz O. C., Taylor R. L., The Finite Element Method - fifth edition, Oxford, Butterworth-Heinemann, 2000. 